\title{
Time variations of IR spectra and dust properties in carbon-rich Mira variables
}

\author{
Hyun-il Sung \\ Korea Astronomy Observatory, 61-1, Whaam-Dong, Youseong-Gu, \\ Taejeon 305-348, Korea \\ Sang-gak Lee \\ Astronomy Program, SEES, Seoul National University, Seoul 151-742, \\ Korea
}

\begin{abstract}
We have studied variations of physical parameters in the circumstellar envelopes of carbon-rich Mira variables at different pulsation phases. After comparing the ISO/SWS spectra of these stars with spectral energy distributions calculated from models, we could find the best-fitted parameters for the physical state of the dust envelope at each phase. The variations of the parameters effective temperature, fraction of $\mathrm{SiC}$ grains, mass-loss rates, and inner radius of shell, are found to have nearly the same trend as that of the light curve. The other parameters grain size, optical depth, radius of central star and dust temperature at the inner boundary of envelope, are inversely varying to the light curve.
\end{abstract}

\section{Introduction}

The presence of dust shells around Mira variables offers an important opportunity to study the effects of atmospheric oscillations on the circumstellar environment. For a few carbon Miras, the variations of line velocity, angular diameter, and effective temperature in the central star were determined by Bergeat \& Sibai (1983) with wide-band photometry. Barnbaum (1992) presented optical radial velocities of carbon Miras with multiple epochs. The pulsation of another carbon Mira, S Cep, has been examined in detail using near-IR spectra by Hinkle \& Barnbaum (1996). The purpose of this study is to investigate the correlations of pulsation phase and dust properties of the carbon-rich Mira variables.

\section{Observations and Data Reduction}

Observations were carried out with the Infrared Space Observatory (ISO) Short Wavelength Spectrometer (SWS; de Graauw et al. 1996) for three carbon-rich Mira variables (Table 1). Optical phases were derived from optical data provided by the American Association of Variable Star Observers (AAVSO). These Miras have long periods with light variations of $\sim 4$ mag in the visual band and large mass-loss rates which were known from the radio observations. The AOT01 
Table 1. Basic data of carbon-rich Mira variables

\begin{tabular}{lccc}
\hline & T Dra & V CrB & V Cyg \\
\hline RA \& Dec & $17: 56: 23.3$ & $15: 49: 31.3$ & $20: 41: 18.3$ \\
(2000.0) & $+58: 13: 06$ & $+39: 34: 18$ & $+48: 08: 29$ \\
Spectral type & C6,2e-8,3e & C6,3e & C7.4e \\
Period (d) & 422 & 421 & 358 \\
Distance (pc) & $525-900$ & 1020 & $580-610$ \\
Visual magnitude & $9-13$ & $7.5-11.5$ & $9-13.5$ \\
\hline \hline
\end{tabular}

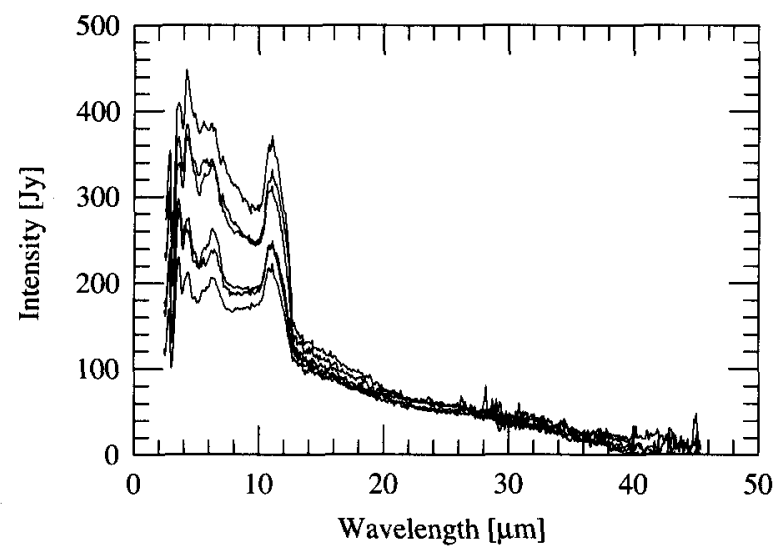

Figure 1. ISO spectra of T Dra. All of the spectra are rebinned. The $11.3 \mu \mathrm{m} \mathrm{SiC} \mathrm{dust} \mathrm{emission} \mathrm{features} \mathrm{are} \mathrm{prominent} \mathrm{and} \mathrm{absorption} \mathrm{bands}$ of $\mathrm{HCN}+\mathrm{C}_{2} \mathrm{H}_{2}$ at $3.1 \mu \mathrm{m}$ and $\mathrm{C}_{3}$ at $5.2 \mu \mathrm{m}$ are strong also. Continuumsubtracted strength of $\mathrm{SiC}$ emission bands increase as light increases.

observing mode was used, which covers the wavelength range between 2.4 to $45 \mu \mathrm{m}$ with a resolution of $\lambda / \Delta \lambda \sim 300-500$.

To reduce the SWS spectra, we used the OSIA and ISAP software. Small discrepancies in flux between different instrumental bands were not corrected except line13 $(\lambda \sim 29-45 \mu \mathrm{m})$. The spectra were then rebinned to one line to fit with the calculated models. The reduced ISO SWS spectra of T Dra, one of three target objects, are shown in Fig. 1.

\section{Radiative transfer models}

In order to model the observed ISO/SWS spectral energy distributions (SEDs) of carbon-rich Mira variables, we performed radiative transfer calculations with the publicly available DUSTY code developed by Ivezić, Nenkova, \& Elitzur (1999). This code is based on exact solutions of a self-consistent equation for the 

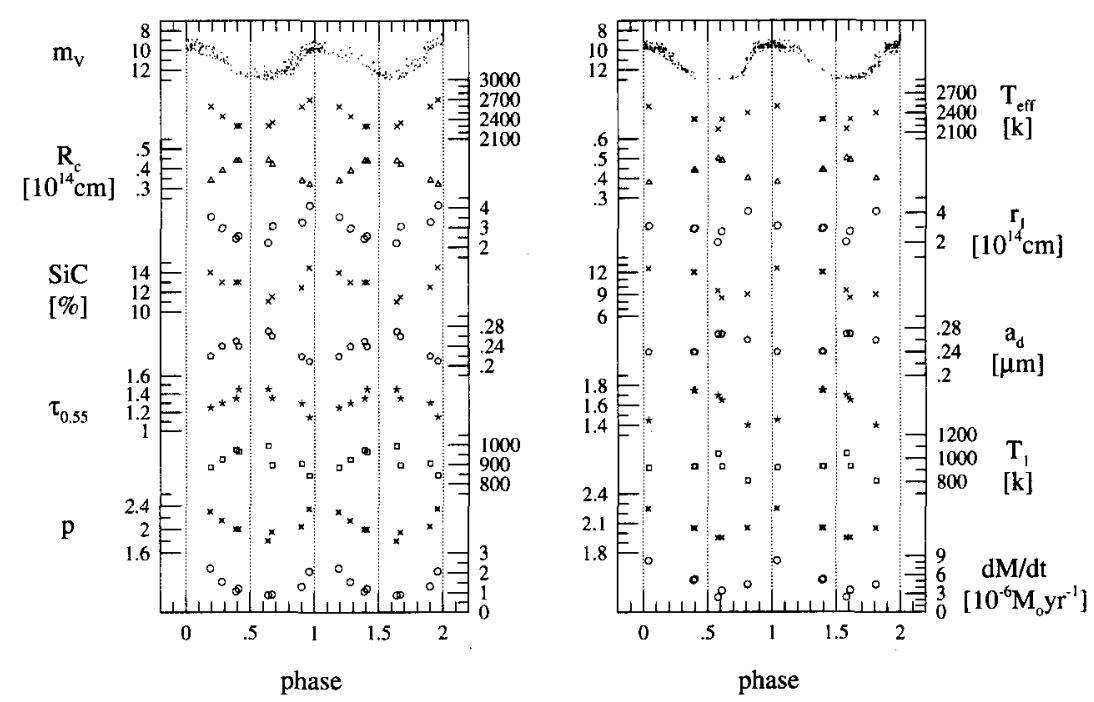

Figure 2. Physical parameters as a function of pulsational phase for carbon-rich Mira variables T Dra and V Cyg.

radiative energy density, including dust scattering, absorption, and emission in spherical shell geometry. It takes full advantage of the scaling properties inherent in the formulation of the problem. The model assumptions, the formulation of the radiative transfer problem, and the scaling properties are described in detail by Ivezić \& Elitzur (1997).

\section{Results and discussion}

The infrared observations of carbon-rich Mira variables at $\sim 6-8$ different epochs and model fits provide variations of physical properties of the circumstellar envelope and dust grains. Fig. 2 shows the optical light curve obtained from the AAVSO and phase dependence of physical parameters determined from model fitting. Each point is plotted twice to emphasize the variability. The maximum and minimum values of physical parameters are presented in Table 2, calculated from the best fitting curves of Fig. 2. Our results are summarized as follows:

1) The variations of effective temperature $\left(T_{\text {eff }}\right)$, inner radius of shell $\left(r_{1}\right)$, fraction of $\mathrm{SiC}$ grains (SiC/AMC ratio) and mass-loss rate $(\dot{M})$ are found to have nearly the same trend as that of light curve. The other parameters: grain size $\left(a_{d}\right)$, optical depth $\left(\tau_{0.55 \mu m}\right)$, radius of central star $\left(R_{c}\right)$ and dust temperature at the inner boundary of envelope $\left(T_{1}\right)$ vary inversely to the light curve.

2 ) The maximum to minimum ratios of $T_{\mathrm{eff}}, \mathrm{SiC} / \mathrm{AMC}, a_{d}, \tau_{0.55 \mu m}$, and $T_{1}$ are more or less around 1.3. In the case of $R_{c}$ and $r_{1}$, these ratios are about 1.5 and 2 , respectively.

3) Mass-loss rates of carbon-rich Miras show large variations, as much as 3.0-3.4 times, during pulsations. The correlation between period and $\dot{M}$ was known, 
Table 2. Values of physical parameters in carbon-rich Miras

\begin{tabular}{lcccccc}
\hline & \multicolumn{2}{c}{ T Dra } & \multicolumn{2}{c}{ V CrB } & \multicolumn{2}{c}{ V Cyg } \\
& Range & Ratio & Range & Ratio & Range & Ratio \\
$T_{\text {eff }}(\mathrm{K})$ & $2250-2800$ & 1.24 & $2400-2900$ & 1.21 & $2100-2550$ & 1.21 \\
$R_{c}\left(10^{14} \mathrm{~cm}\right)$ & $0.31-0.47$ & 1.52 & $0.25-0.40$ & 1.60 & $0.37-0.50$ & 1.35 \\
$r_{1}\left(10^{14} \mathrm{~cm}\right)$ & $2.2-4.3$ & 1.95 & $1.0-2.5$ & 2.50 & $2.1-4.1$ & 1.95 \\
$\mathrm{SiC} \mathrm{ratio}^{1}$ & $0.11-0.145$ & 1.32 & $0.16-0.22$ & 1.38 & $0.08-0.13$ & 1.63 \\
$a_{d}(\mu \mathrm{m})$ & $0.21-0.28$ & 1.33 & $0.25-0.32$ & 1.28 & $0.24-0.28$ & 1.17 \\
$\tau_{0.55 \mu \mathrm{m}}$ & $1.1-1.5$ & 1.36 & $0.6-1.15$ & 1.92 & $1.4-1.8$ & 1.29 \\
$T_{1}(\mathrm{~K})$ & $830-1000$ & 1.20 & $1000-1300$ & 1.30 & $800-1050$ & 1.31 \\
$p$ & $1.8-2.4$ & 1.33 & $1.8-2.4$ & 1.33 & $1.9-2.2$ & 1.16 \\
$\dot{M}$ & $0.7-2.4$ & 3.43 & $0.7-2.1$ & 3.00 & $2.5-8.0$ & 3.20 \\
$\left(10^{-6} \mathrm{M}_{\odot} / \mathrm{yr}\right)$ & & & & & & \\
\hline \hline
\end{tabular}

${ }^{1} \mathrm{SiC} /(\mathrm{SiC}+\mathrm{AMC})$ ratio

and it is supposed that the large values of maximum to minimum ratios of $\dot{M}$ during pulsations were made the scatter in that correlations.

4) The variation of grain size follows the change of two rates, the evaporation rate (by the stellar wind) and the growth rate (by the coagulation), which varies with $T_{\text {eff. }}$. We found that the minimum size of grains at maximum light, and maximum size of grains at minimum light, as expected.

5) The inner boundary of the dust shell, $r_{1}$, and the dust temperature at the inner boundary, $T_{1}$, were inversely variable, i.e. $T_{1}$ become a maximum at the minimum of $r_{1}$, because $T_{1}$ was not dependent only on distance from the central star but was proportional to the other physical parameters in the form of $T_{1} \propto T_{\text {eff }}\left(R_{c} / r_{1}\right)^{1 / 2}$.

6) The slope of the SED at long wavelengths $(\lambda>15 \mu \mathrm{m})$ as shown in Fig. 1 was dependent the index $p$ of the power-law density distribution.

\section{References}

Barnbaum, C. 1992, AJ, 104, 1585

Bergeat, J., Sibai, A.M. 1983, A\&A, 119, 207

de Graauw, T., et al. 1996, A\&A, 315, L49

Hinkle, K.H., Barnbaum, C. 1996, AJ, 111, 913

Ivezić, Ž., Elitzur, M. 1997, MNRAS, 287, 799

Ivezić, Ž., Nenkova, M., Elitzur, M. 1999, User Manual for DUSTY, University of Kentucky Internal Report, http://www.pa.uky.edu/ moshe/dusty 